\title{
Pragmática intercultural, social y cognitiva
}

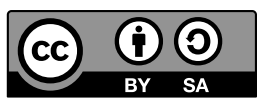

\author{
«What do people do when using language?» \\ Jef Verschueren
}

Cuando Charles Morris (1938: 6) definió la pragmática como la ciencia que estudia «the relation of signs to their interpreters ${ }^{1} »$ acuñó el término en el sentido que le damos hoy y, al mismo tiempo, empezó con el debate sobre la relación que guarda la pragmática con otras disciplinas. Según Morris, la pragmática, junto con la sintaxis y la semántica, forma parte de una ciencia general de los signos, llamada semiótica. Si la sintaxis se centra en las relaciones formales entre los signos, y la semántica en las relaciones entre los signos y los objetos a los que los signos se refieren, la pragmática, siempre según Morris, estudia las relaciones entre las expresiones lingüísticas, sus significados y los usos concretos en diferentes contextos. La pragmática se aproxima así considerablemente a la semántica e incluso en algunos estudios se considera solo el apéndice de ella. Sin embargo, pronto empezó a irse consolidando como una disciplina independiente, más aún cuando Paul Grice $(1957,1975)^{2}$ y sus seguidores ubican a los usuarios del lenguaje en el mismo centro de la teoría e insisten en que el significado pragmático es el así llamado significado del hablante. Aunque hoy en día la pragmática sí se considera una disciplina autónoma, todavía hay diferentes maneras de entender el campo de su investigación: por una parte, está integrada en la lingüística, pero por otra, se entiende como una nueva perspectiva de análisis que debe aplicarse al estudio de todos los fenómenos lingüísticos. Uno de los representantes clave de este último acercamiento es Jef Verschueren, que define la pragmática como «the cognitive, social, and cultural science of language and communication» (Verschueren, 1994: 1) ${ }^{3}$. De manera

1 Charles Morris (1938): «Foundations of the Theory of Signs». En: Otto Neurath, Rudolf Carnap and Charles Morris (eds.) International Encyclopedia of Unified Science. Chicago: University of Chicago Press, 77-138.

2 Paul Grice (1957): «Meaning», Pbilosopbical Review, 66(3); Paul Grice (1975): «Logic and Conversation», Syntax and Semantics, vol.3 edited by P. Cole and J. Morgan, Academic Press. Jef Verschueren (1999): Understanding Pragmatics. London: Edward Arnold LTD. 
parecida Jacob Mey (2001: 5$)^{4}$ insiste en que la pragmática estudia «the use of language in buman communication as determined by the conditions of society».

Sea cual sea la línea principal de la investigación pragmática, es obvio que tal estudio debe abarcar el lenguaje en su funcionamiento, es decir, tener en cuenta a todos los factores que rodean al objeto lingüístico en su producción, y solo así será posible responder a la pregunta que se plantea Verschueren (1999): qué hace la gente cuando usa el lenguaje.

Nos interesa, pues, cómo se producen, cómo se transmiten, qué motivación tienen y cómo se interpretan los significados, lo que implica tener en cuenta tanto los factores individuales y cognitivos como los factores diastráticos, diafásicos y diatópicos. En cuanto a esto, se pueden diferenciar dos posturas en los análisis: un acercamiento de aspecto cognitivo-filosófico que se centra en la expresión del significado, en la transmisión de la información y en la parte cognitiva del uso del lenguaje ${ }^{5}$, y otro posicionamiento que destaca el aspecto más antropológico, es decir, la necesidad de tomar en consideración tanto a los usuarios del lenguaje como al entorno y a las condiciones sociales y culturales ${ }^{6}$ en que se produce el mensaje. En la actualidad aparecen algunas propuestas que pretenden integrar los enfoques, tanto el cognitivo como el socio-cultural, por ejemplo, la de Istvan Kecskes $(2014)^{7}$, lingüista estadounidense y padre de la pragmática intercultural. En su obra, Intercultural Pragmatics, ofrece una nueva perspectiva socio-cognitiva que estudia la manera de cómo se entrelazan los factores individuales y culturales en la comunicación, centrándose en la interacción social a través de las líneas culturales y en la forma en que se usa el lenguaje en estas interacciones. Por otro lado, los intentos de incluir los factores sociales y culturales en el análisis pragmático no son del todo nuevos, ya que, por ejemplo, las teorías principales sobre la cortesía de Robin Lakoff (1973) ${ }^{8}$, Geofreey Leech $(1977)^{9}$, Penelope Brown y Stephen Levinson $(1978,1987)^{10}$ parten del prin-

4 Jacob Mey (2001): Pragmatics: An Introduction. 2nda edición. Oxford: Blackwell.

5 Véase Austin (1962); Grice (1967), Searle (1969), Brown y Levinson (1983, 1987) Sperber and Wilson (1986).

6 Como por ejemplo Verschueren (1999) y Mey (1993).

7 Istvan Kecskes (2014): Intercultural Pragmatics. New York: Oxford University Press.

8 Robin Lakoff (1973) «The logic of politeness». En: C. Corum, T. Cedric Smith-Stark, A. Weiser (eds.): Papers from the Ninth Regional Meeting of the Chicago Linguistics Society, Chicago: Department of Linguistics, University of Chicago, 292-305.

9 Geofreey Leech (1977): Language and Tact. Trier: Linguistic Agency, University of Trier.

10 Penelope Brown y Stephen Levinson (1987): Politeness. Cambridge: Cambridge University Press. 
cipio cooperativo de Grice y lo complementan, precisando los factores sociales y culturales imprescindibles para que el acto cortés pueda tener lugar.

En la actualidad siguen existiendo divergencias teóricas en cuanto a la pragmática y su relación con otras disciplinas, así como en lo que atañe a diferentes enfoques del análisis pragmático. Como creemos que tanto los factores cognitivos como los sociales y culturales son constituyentes contextuales relevantes a la hora de estudiar un proceso comunicativo, hemos querido recoger en este número temático, La pragmática intercultural, social y cognitiva, investigaciones con enfoques teóricos y metodológicos circunscritos en todos estos ámbitos, a saber, la pragmática intercultural, pragmática social, pragmática poética, pragmática lingüística y pragmática cognitiva. Los estudios seleccionados para este volumen se articulan en tres secciones principales: lingüística, literatura y didáctica.

El capítulo de la lingüística consta de nueve estudios. Es un honor poder contar en primer lugar con la investigación de María Victoria Escandell Vidal quien, en su artículo «El futuro simple del español. Sistema natural frente a usos cultivados», pone de relieve la complejidad del futuro simple en español debido a su aparente carácter polisémico y presenta un enfoque unificador que aúna los usos conjeturales y prospectivos para plantear una propuesta monosémica donde el futuro quepa bajo una semántica de tipo evidencial. Le sigue el estudio de Marek Baran, «La emotividad y la tipificación sociopragmática de los ethnolectos», quien se sitúa en el ámbito de los estudios de la cortesía verbal, la etnografía de la comunicación y la pragmática intercultural para explicar el aspecto sociopragmático de la emotividad, a partir de la perspectiva etnometodológica, basada en el concepto del ethos comunicativo y de los etholectos conversacionales. El tercer estudio, «Cortesía y grado de fuerza en la realización de los 'actos amenazadores de la imagen' en español», de Nuria Campos Carrasco, propone una clasificación de las estructuras utilizadas para la expresión de los actos de habla ilocutivos directivos enmarcados dentro de la cortesía y grado de fuerza en la realización de los actos amenazadores de la imagen conminatorios en español. Rosa Martín Gascueña, por su parte, se acerca a la pragmática de la cortesía en su artículo «Cortesía en el inicio y el final de los discursos de aceptación del Premio Cervantes», utilizando como corpus todos los discursos de aceptación del premio Cervantes hasta 2017 para proporcionar posibles rasgos de la idiosincrasia e ideología de los premiados basándose tanto en variaciones diacrónicas significativas como en variables socio-culturales. En el quinto artículo, «Los artículos definido e indefinido 
del español (o castellano) en contexto», Sonia Montero Gálvez parte de la pragmática cognitiva para enfrentarse al problemático tema del contraste entre el artículo definido e indefinido y ofrece una conceptualización referencial centrada en la delimitación inclusiva o exclusiva de una cantidad delimitada, rompiendo así con la visión tradicional del artículo. Le sigue el estudio de María Pilar Sanchis Cerdán, «Estudio sobre el uso del marcador discursivo para empezar: identificación de valores», que investiga el marcador discursivo para empezar desde un enfoque pragmático y apunta al valor epistémico como uno de los empleos más numerosos instando a una redefinición lingüística de este marcador. En «Usos de la deixis espacial en muestras orales de hablantes de la ciudad de Santiago de Cuba. El caso del adverbio demostrativo aqui» Tania Ulloa Casaña aborda desde una perspectiva pragmática otros usos discursivos de la expresión deíctica aquí, alejados de su uso común, utilizando como corpus muestras orales de hablantes de Santiago de Cuba. En el penúltimo estudio en este apartado, «El foco en español: problemas de definición y manifestaciones sintácticas», Vita Veselko aborda el complejo tema del foco en español y propone por un lado una redefinición del término junto a sus rasgos caracterizadores, y por el otro examina la relación de la función informativa del foco con el grado de novedad de la información, así como su correspondencia con la noción de contraste. Por último, cierra esta sección el artículo de Andrzej Zieliński, «Cognición y sociedad. Los tratamientos en los albores del feudalismo: señor y las fórmulas honoríficas con -ísimo», que se remonta a través de un estudio cognitivo diacrónico al origen de las fórmulas de tratamiento señor y las fórmulas honoríficas con -ísimo rastreando su evolución dentro de las implicaciones del feudalismo como nuevo modelo social.

El apartado de literatura consta de dos investigaciones. En la primera, «Elementos para un análisis cognitivo del discurso poético», Ángel Luján Atienza, parte de la poética cognitiva y la pragmática poética y propone la aplicación de diferentes herramientas analíticas para un análisis cognitivo del discurso poético a nivel oracional, textual y discursivo con el objetivo de crear un acercamiento global a la poesía basado en el principio de relevancia. Se le une el artículo en portugués, «Análise semântico-pragmática do futuro na tragédia $A$ Castro», de las investigadoras eslovenas Mojca Medvedšek y Blažka Müller Pograjc, quienes remiten a la tragedia de António Ferreira, $A$ Castro, para presentar desde un análisis semántico-pragmático las características y el funcionamento de los actos ilocutorios enmarcados en la esfera del futuro. 
El volumen temático se cierra con una investigación en la sección de didáctica titulada « ¿Estandarizar lo diferente? El papel de los estándares de la competencia intercultural en la educación formal», en donde Ana Jovanović crea un camino de acercamiento a la evaluación de la competencia comunicativa intercultural, delimitando los aspectos más sensibles (como la descripción objetiva de criterios de evaluación, si es objetivamente posible su evaluación o el aspecto moral que todo ello conlleva). Desde una metodología autoetnográfica, informa en primera persona de un proyecto piloto llevado a cabo en Serbia en el que se intentó definir los estándares de competencia para las lenguas en la educación primaria.

Esperamos que todos los artículos que componen la polifonía de propuestas y perspectivas del volumen La pragmática intercultural, social y cognitiva susciten el interés suficiente como para seguir tejiendo nuevas investigaciones y retos que respondan a todas las preguntas abiertas sobre el lenguaje y el contexto planteadas en este número.

Barbara Pihler Ciglič, Gemma Santiago Alonso 